LCLS-TN-06-15

September 2006

\title{
Cold Test Measurements on the GTF Prototype RF Gun ${ }^{\dagger}$
}

\author{
S.M. Gierman and J.F. Schmerge
}

\begin{abstract}
The SSRL Gun Test Facility (GTF) was built to develop a high brightness electron injector for the LCLS and has been operational since 1996. Based on longitudinal phase space measurements showing a correlated energy spread the gun was removed and recharacterized in 2002. The low power RF measurements performed on the gun are described below. Perturbative bead measurements were performed to determine the field ratio in the two-cell gun, and network analyzer measurements were made to characterize the mode structure. A second probe was installed to monitor the RF field in the first cell, and a diagnostic was developed to monitor the high-power field ratio. Calibration of the RF probes, a model for analyzing RF measurements, and Superfish simulations of bead and RF measurements are described.
\end{abstract}

\section{Introduction}

The GTF photo-injector gun is an S-band standing-wave structure, with two resonant cavities and an intervening thick washer (Fig. 1). The flat, back wall of the first cavity is a copper plate without penetration that serves as photocathode when illuminated with ultraviolet light from a pulsed, high-power laser. RF power enters the gun through an iris on the outer wall of the second cavity, and is coupled to the first through the axial opening of the washer. The first cavity is often referred to as a half cell, because its fullcell length has been truncated by the cathode plate. The second cavity is simply the full cell. The photo-injector gun produces bunched electrons with up to $6.5 \mathrm{MeV}$ of energy.

The gun is designed to operate in a balanced $\pi$ mode, one in which the maximum standing-wave amplitude for the axial electric field is the same in both cells. When first commissioned, field amplitude was manipulated by a pair of insertion rods in the outer wall of the full cell. A threaded rod was later attached to the back surface of the cathode plate, allowing the field to be tuned by a plunging action applied to a central portion of the cathode (which is constrained not to move at its outer edge). Slater-type perturbative bead methods were used for field measurement during the initial commissioning. Since bead measurements cannot be performed in situ, subsequent tunings (such as after the installation of a new cathode) relied on mode separation as an indicator of field balance. The first fundamental mode in the gun is a zero mode. When mechanically tuned for field balance, the $\pi$ mode is overcoupled, the zero mode undercoupled, and the two are separated by $3.50 \mathrm{MHz}$. The gun can therefore be tuned online using a network analyzer and reflected power measurements. The procedure is to first tune for field balance, and

\footnotetext{
${ }^{\dagger}$ Work supported in part by DOE contract DE-AC02-76SF00515.

This work was performed in support of the LCLS project at SLAC.
} 
then to match the $\pi$-mode resonance to the high-power drive by an adjustment to the operating temperature (cooling water) of the gun.

This note describes a second round of bead and RF measurements that were made during the installation of a field probe for the half cell. Together with an existing full-cell probe, the two probes constitute a diagnostic for monitoring the field ratio during highpower operations. ${ }^{\dagger}$ Since these were the first bead measurements after the installation of the cathode-plate tuning rod, the mode structure of the gun was explored over a relatively wide range of cathode displacements. At each displacement, an axial field profile for each mode was obtained with bead measurements, and network analyzer measurements of reflected power and probe response were acquired as frequency-space waveforms.
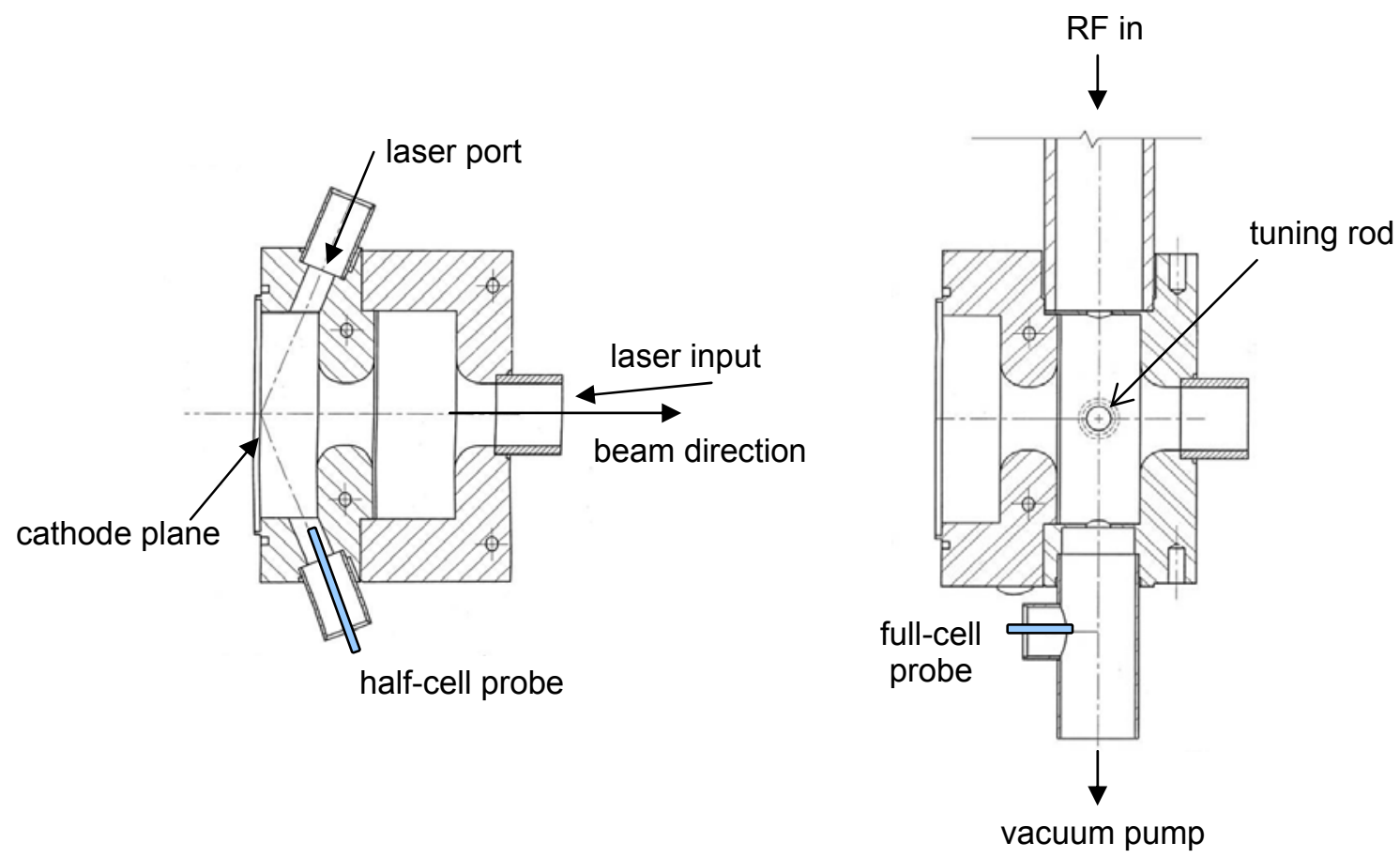

Figure 1 Two cross sections of the GTF gun, related by a 45 degree axial rotation. The cathode plate that makes the back wall of the half cell is not shown. Laser light for photo-emission enters either through the side port on the half cell or from downstream.

\section{Bead measurements}

The physical basis for the measurement of cavity fields by the insertion of beads or other small objects, and for the cavity tuning methods described above, resides in the Slater perturbation theorem, ${ }^{1}$ which for small perturbation states that the fractional change in resonant frequency effected by the object or perturbed boundary is proportional to the fractional difference in stored magnetic and electrical energy at the perturbed location, ${ }^{2}$

\footnotetext{
${ }^{\dagger}$ By field ratio we mean the ratio of $\pi$-mode standing-wave maxima for the axial electric field in the two cells, which is unity for a balanced gun. The maximum in the half cell occurs at the cathode, and in the full cell near its center.
} 


$$
\frac{\omega-\omega_{0}}{\omega_{0}}=\frac{k \int_{\Delta v}\left(\mu H^{2}-\varepsilon E^{2}\right) d v}{4 U} .
$$

Here $\omega_{0}$ is the unperturbed (angular) frequency, and $H$ and $E$ are the unperturbed magnetic and electric fields. The integral is over the volume removed, and $U$ is the timeaveraged stored energy. The constant $k$ depends upon the shape of the perturbing volume. Applied to the measurement of an electric field profile on the axis of the S-band gun, the theorem states that a short, needle-like object will produce a change in resonant frequency proportional to the square of the local electric field (since the magnetic field vanishes on axis). Since our concern is relative rather than absolute field strength, the value of the constant of proportionality is irrelevant.

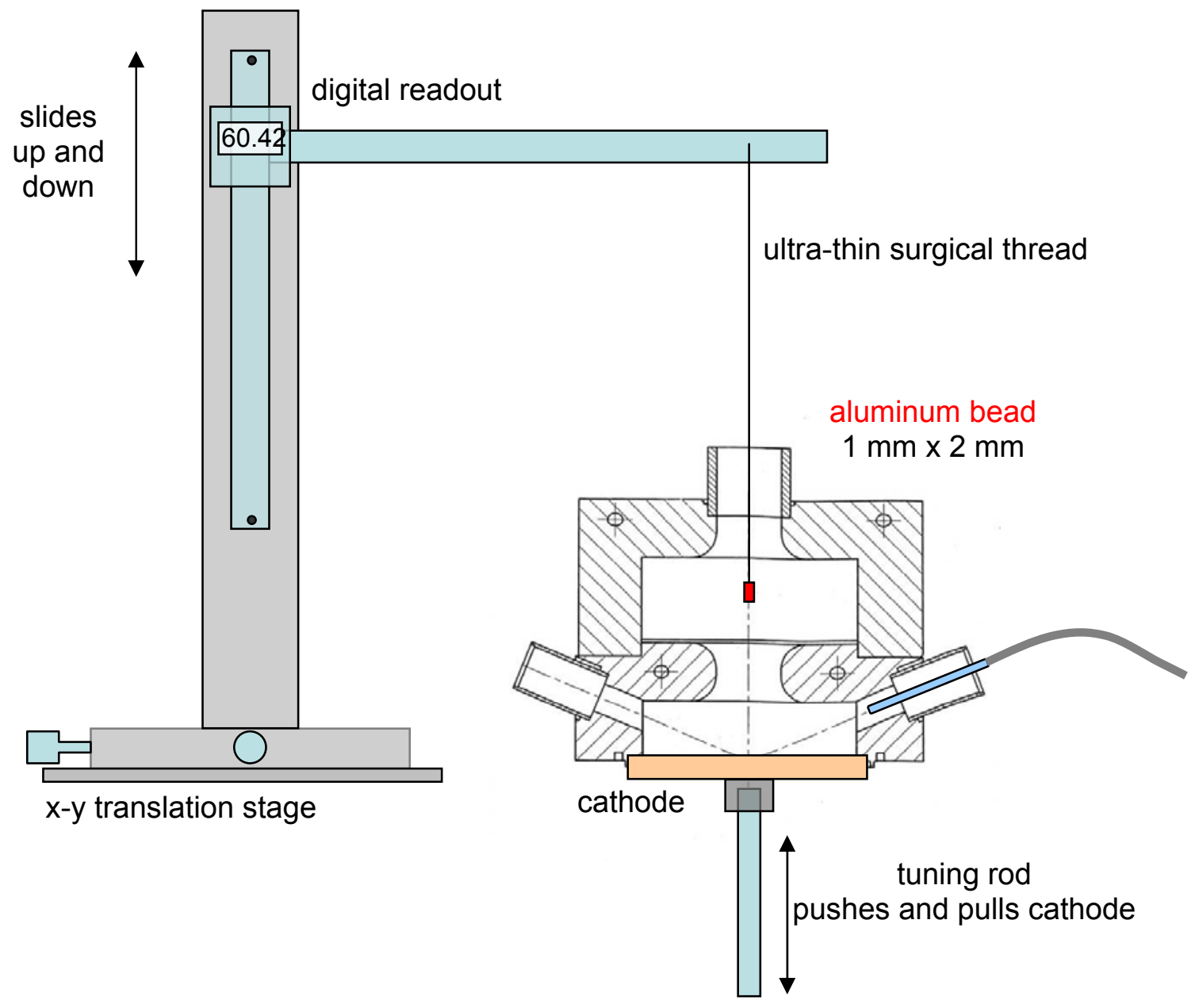

Figure 2 Setup for bead measurements. Network analyzer connections are shown in Fig. 3.

Our setup for bead measurements is illustrated in Fig. 2. The gun was mounted in a vertical direction, and a suspended bead lowered into it by means of a sliding rail. After some experimentation, which consisted of measuring the change in resonant frequency produced by several different beads and suspension materials, we settled on a 
cylindrically shaped aluminum bead $1 \mathrm{~mm}$ in diameter and $2 \mathrm{~mm}$ in length, suspended on a single strand of ultra-thin surgical thread. This arrangement produced a $150 \mathrm{kHz}$ shift in the $\pi$-mode resonance of the tuned gun. Rejected for their smaller frequency shifts were similarly sized, non-conducting Delrin beads. Preferring a stiffer suspender, we experimented with stripping the outer layers of fiber-optic cables and using the inner cores, but it was difficult to produce these uniformly so each piece made its own unique contribution to the frequency shift, which had to be accounted for by repeating the lengthy measurements with the bead unattached. The advantage of the surgical thread was that it had no measurable effect on frequency, thereby cutting in half the measurement time. A disadvantage was that the small bead did not fully load the thread for straightness, and the measurement environment had to be isolated from air currents that could sway the position of the bead. Technical challenges included the bead/thread attachment, made by glue: given the insubstantial thread and viscous glue, it was again difficult to make straight the orientation of the suspended cylinder.

Profiles of axial electric field require measurement of resonant frequency vs. bead position. ${ }^{3}$ Attached to the sliding rail was a position indicator with 10 micron resolution. An HP $8753 \mathrm{C}$ network analyzer was used for frequency measurement. RF power from the analyzer was coupled to the gun through the high-power waveguide connected to the full cell (Fig. 3). At each bead position the analyzer swept the drive frequency and identified as resonant the frequency that minimized reflected power. Also recorded was the reflection coefficient at resonance. By decoupling and exciting independently the two channels of the network analyzer, we obtained simultaneous measurements of the zeroand $\pi$-mode resonances, each with a resolution near $1 \mathrm{kHz}$. An ultimate resolution closer to $5 \mathrm{kHz}$ resulted from the temperature dependence of the resonant frequency, approximately $-52 \mathrm{kHz} / \mathrm{C}$ for the GTF gun. Although temperature was recorded as a function of bead position, the $0.1 \mathrm{C}$ resolution of the thermocouple was too coarse to allow correction, as this was also the magnitude of typical deviations during the twenty minutes required to complete a profile. Data collection via GPIB was computer controlled and displayed in real time using LabView, except for bead position which had to be adjusted manually, and the value from the digital readout had then to be entered into the control panel. 


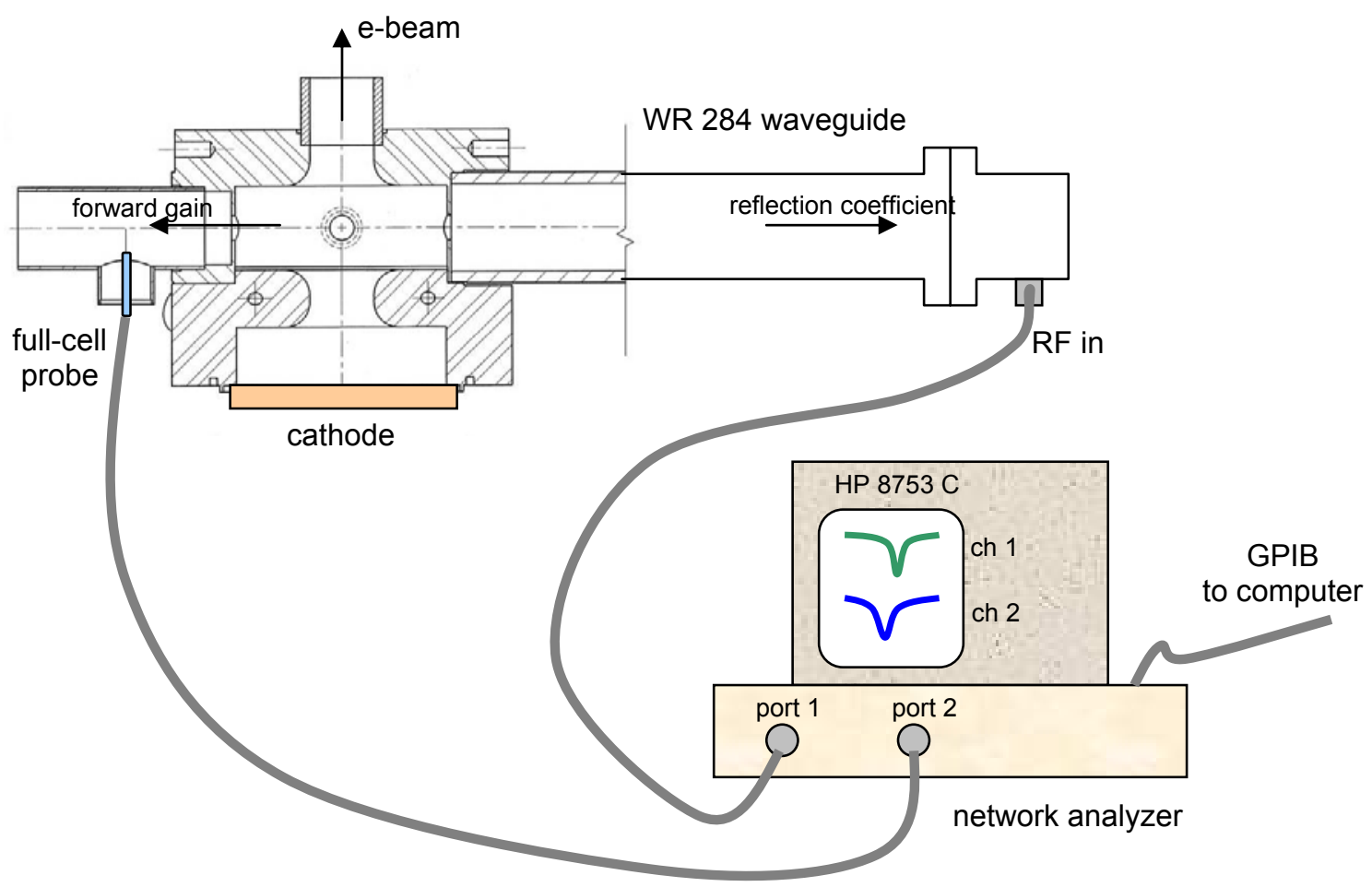

Figure 3 Network analyzer connections to gun. Port 1 connects to the high-power waveguide that feeds the full cell, and port 2 connects to either the full-cell or half-cell probe. Bead measurements require only reflection measurements $S_{11}$, but transmission measurements $S_{21}$ to each probe were also acquired for each configuration of the cathode tuning plate.

A representative bead scan is shown in Fig. 4. Bead measurements start outside the gun in the fringe field, and continue until the bead almost touches the cathode. The field ratio for the case shown is 1.18 (see caption). A summary in terms of $\pi$-mode frequency perturbation and field ratio for a set of eight coarse tunings (cathode displacements) is given in Fig. 5. The field ratio $E_{2} / E_{1}$ ranged from 1.74 to 0.64 as the cathode plate was withdrawn from the half cell. The differential screw mechanism that drives cathode displacement is engineered for 141 microns per revolution, but there is significant backlash when changing directions. A dial indicator attached to an external flange registered a 240 micron displacement over all measurements. Simulations suggest a displacement not less than 166 microns. 


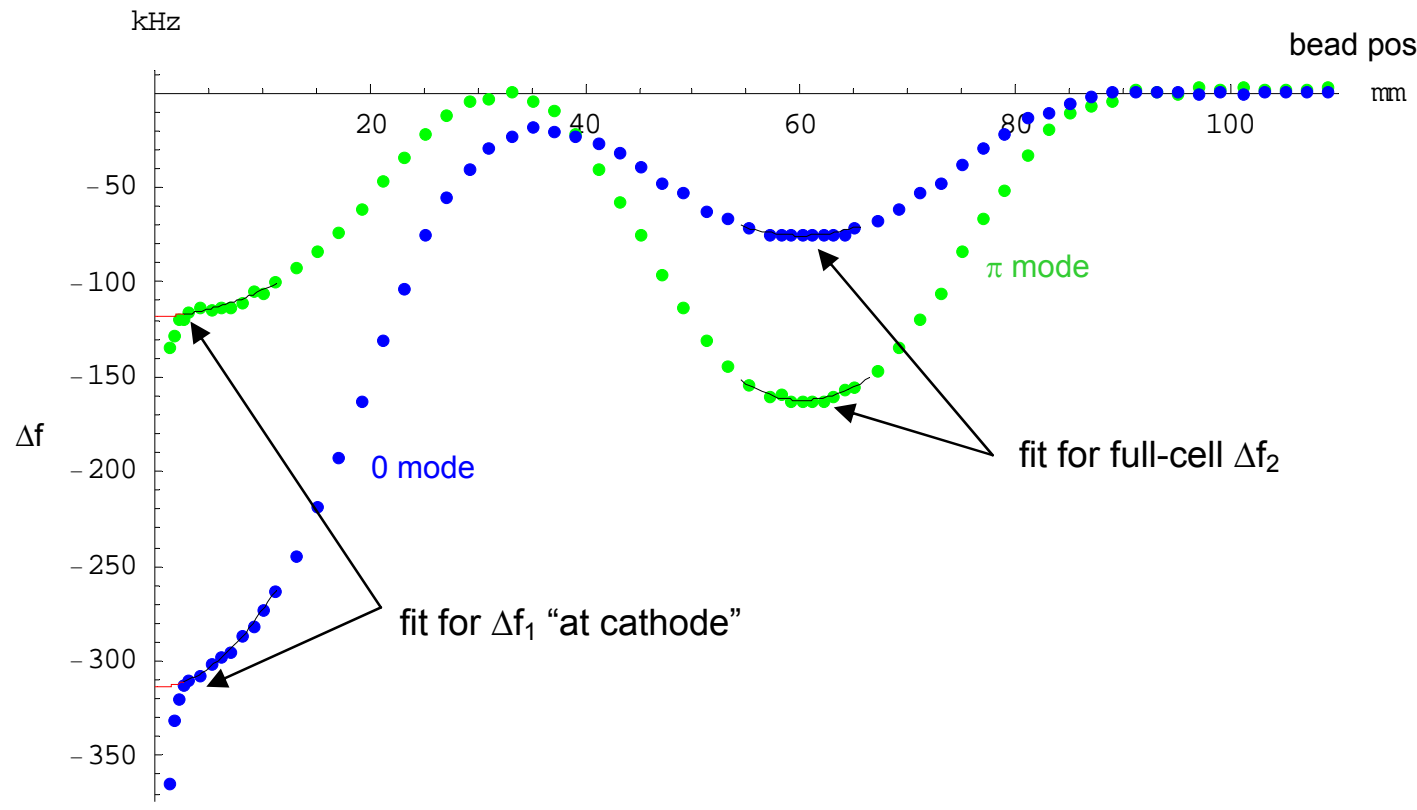

tune 3

Figure 4 Sample bead scan, third of eight gun tunings. Shown is the measured change in resonant frequency $\Delta \mathrm{f}$ in $\mathrm{kHz}$ vs. bead position in $\mathrm{mm}$ for the zero and $\pi$ modes. The cathode is at the left side of the plot. The profiles are proportional to the square of the axial electric field. Maximum values $\Delta \mathrm{f}_{2}$ in the full cell are selected by a local quadratic fit (solid line). A similar fit is made for $\Delta \mathrm{f}_{1}$ "at the cathode", excluding points nearest the cathode where $\Delta \mathrm{f}$ runs away, and constraining the fit to have zero derivative at the cathode surface. The measured $(\pi$ mode $)$ field ratio is $E_{2} / E_{1}=\left(\Delta f_{2} / \Delta f_{1}\right)^{1 / 2}=(164 / 118)^{1 / 2}=1.18$.
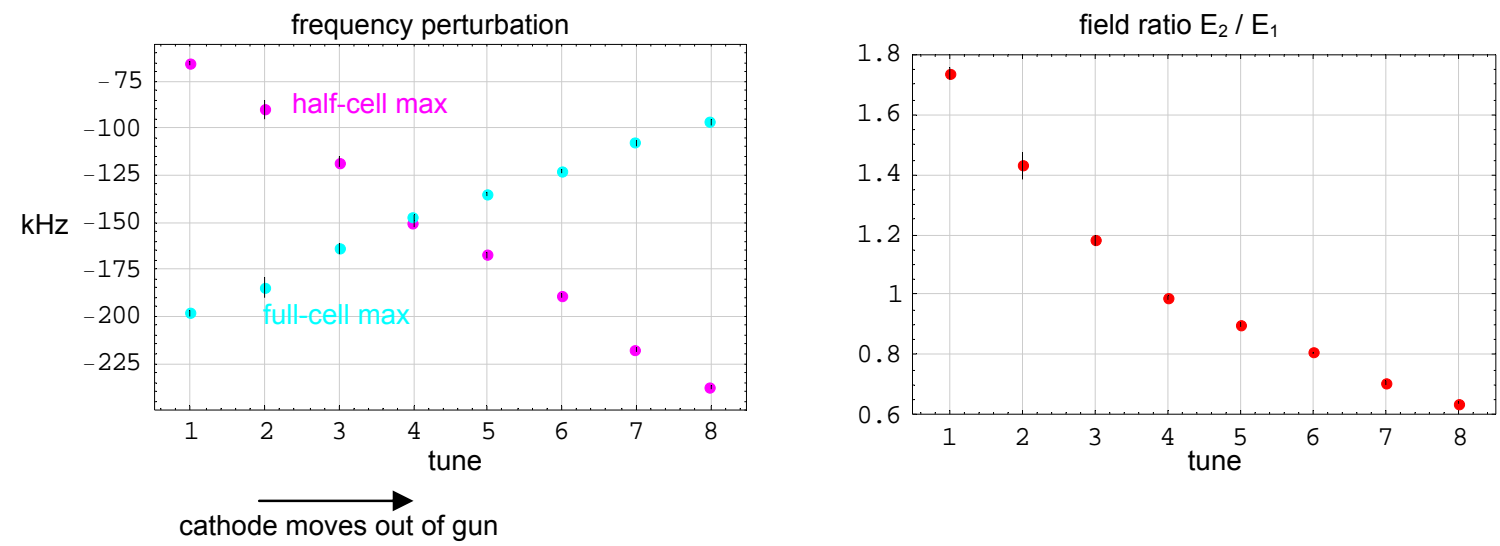

Figure 5 Summary of bead measurements, coarse tuning, $\pi$ mode only. The horizontal axis is a list representing eight gun tunings, ordered in terms of cathode displacement. As the cathode is withdrawn from the gun, the bead makes a larger perturbation in the half cell (field increases at the cathode) and a smaller one in the full cell (field decreases). The fourth tuning is near field balance. The field ratio $E_{2} / E_{1}$ ranges from 1.74 to 0.64 . 


\section{Simulated bead measurements}

For comparison with code models, bead measurements were simulated with the twodimensional field solver POISSON SUPERFISH. ${ }^{4}$ Because it is a 2 -D code, not all aspects of gun geometry (such as insertion rods and other cavity penetrations) can be accounted for in a direct way; instead, our model used measured resonant frequencies, with and without the cathode plate attached, to adjust the diameters of the two cavities. Cavity tuning was modeled by holding fixed the outer diameter of the cathode plate, and displacing linearly the area approaching a central area with uniform displacement. The diameter of the central displacement was chosen to match that of the applied force, which turned out to be close to the one that achieves maximum frequency perturbation (for larger diameters the increasing magnetic field lessens the perturbation). Cathode displacement was chosen to match the simulated field ratio to the measured value, and the resulting frequency perturbations were then checked for consistency with the simulated field profile, and for agreement with the measured perturbations. Figure 6 shows a simulated bead scan, and compares frequency perturbations with field values. The comparison with measurement is given in Fig. 7, including a comparison of predicted and measured mode separation for the set of eight gun tunings.
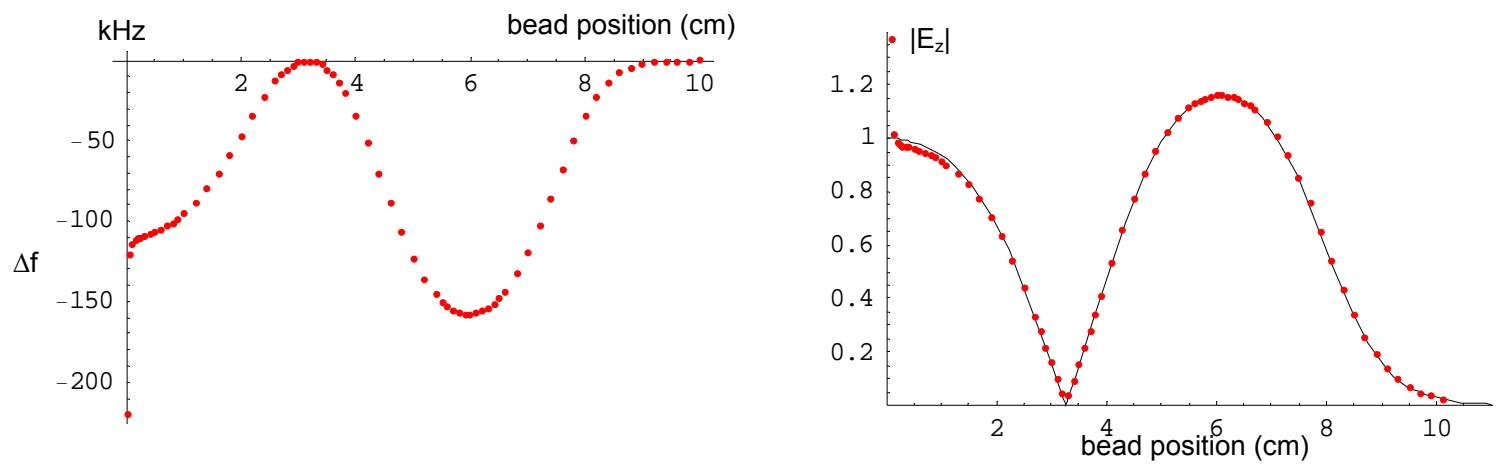

Figure 6 Superfish simulation of bead scan. The plot on the left shows frequency perturbation vs. bead position (note the upward run very near the cathode). On the right, the solid line is the Superfish electric field (absolute value) in the absence of the bead, normalized for unity at the cathode. The square root of the frequency perturbations have been multiplied by a constant of proportionality chosen to match the field maximum in the second cell. The good match on the remainder of the curve validates the choice of bead and the interpretation of frequency perturbation as an indicator of field strength. In some cases there is a small error approaching the cathode. 

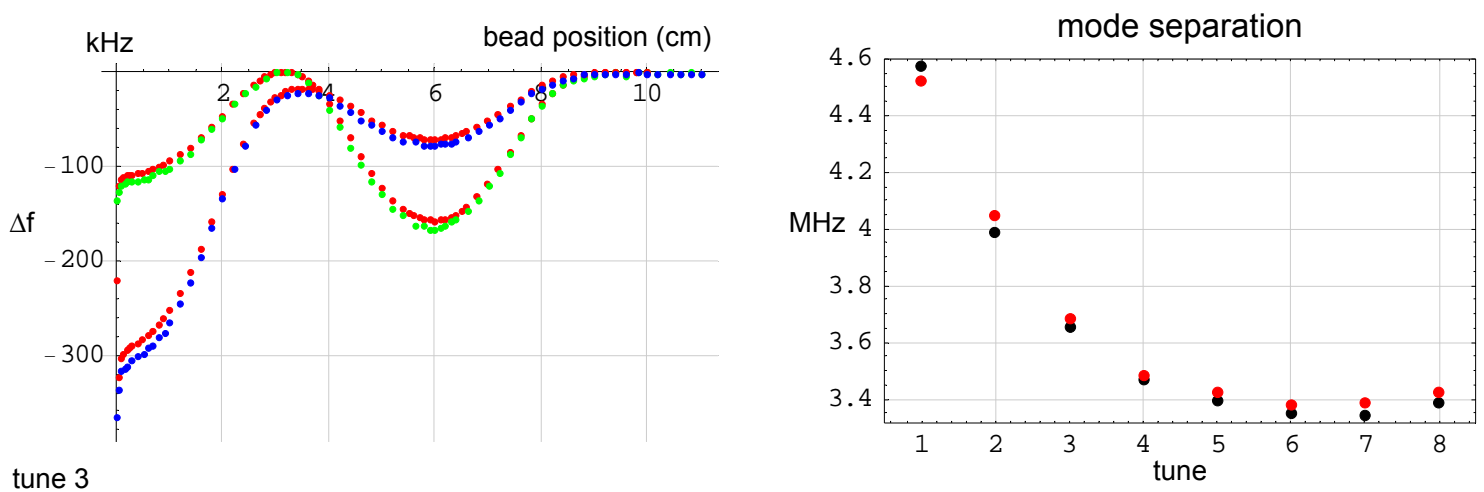

tune 3

Figure 7 The left plot shows frequency perturbations for the third gun tuning, with $\pi$-mode measurements in green, zero-mode measurements in blue, and Superfish simulation in red. For the set of eight gun tunings, the right plot shows mode separation $\left(\mathrm{f}_{\pi}-\mathrm{f}_{0}\right)$ as measured (black) and simulated (red).

\section{Other RF measurements}

For each displacement of the cathode plate, the frequency response of the gun was measured with a network analyzer. Measured were the reflection coefficient $\mathrm{S}_{11}$, and the transmission coefficient $\mathrm{S}_{21}$ (forward gain) of each of the two field probes. The results are summarized in Fig. 8, which shows the magnitude of the reflection coefficient and the loaded Qs for each of the modes. The ratio of the magnitudes of the full- and half-cell transmission coefficients, evaluated at the resonant frequency of the $\pi$ mode, are fit to the field-ratio bead measurements in Fig. 9. Sample waveforms are given in the next section.
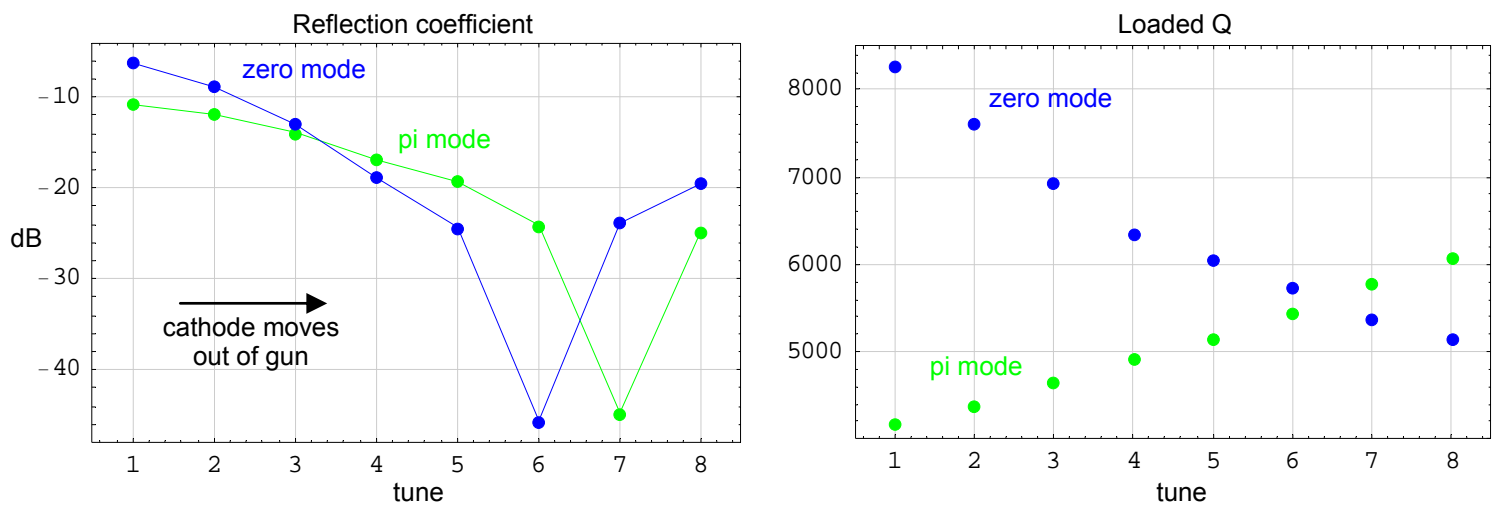

Figure 8 Mode structure as characterized by reflection coefficient and loaded Q. The left plot shows the zero mode near critical coupling at tune 6 , and the $\pi$ mode at tune 7 . From the phase of the reflection coefficient (not shown), it is observed that the zero mode is undercoupled and the $\pi$ mode overcoupled for tunes to the left of critical coupling, and vice versa for tunes to the right. The loaded Q (right plot) was derived from half-power resonant bandwidths measured with the field probes. Minimum mode separation likely occurs between tunes 6 and 7 (see Fig. 7). Recall that $\pi$-mode field balance occurs at tune 4 . 


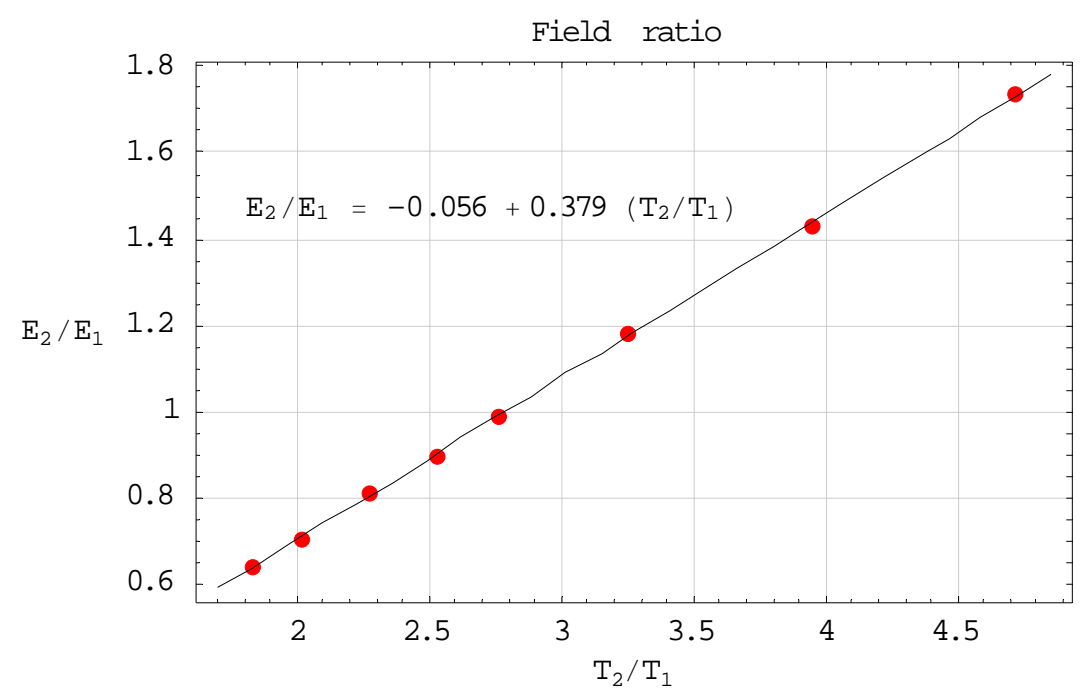

Figure 9 Field ratio as a function of probe ratio. Here the subscripts 1 and 2 stand for half- and full-cell values. $T=\left|S_{21}\right|$ is the magnitude of the transmission coefficient, $E_{2} / E_{1}$ is the field ratio from the bead measurements.

\section{Model for RF measurements}

The RF probes are antennas made from coaxial line with the center conductor extended past the outer conductor. Each probe has a different gain, which was controlled by adjusting the length of the conductor. Figure 10 shows the frequency response $S_{21}$ of both probes after tuning the gun for field balance. The constant and linear terms of the measured phase have been modified to correct the phase reference point of the network analyzer and path-length differences. Note that the mode at $2856 \mathrm{MHz}$ has a $180^{\circ}$ phase shift between cells while the remaining mode has a $0^{\circ}$ phase shift. These are by definition the $\pi$ and zero modes respectively.

A model of the two-cell gun composed of two bandpass filters was used to fit the data with a least square error fitting routine. The $S_{21}$ waveforms were fit to the response function

$$
T(f)=\frac{A_{\pi}}{j Q_{\pi}\left[\left(f / f_{\pi}\right)-\left(f_{\pi} / f\right)\right]+1}+\frac{A_{0}}{j Q_{0}\left[\left(f / f_{0}\right)-\left(f_{0} / f\right)\right]+1},
$$

where $f_{\pi}$ and $f_{0}$ are the resonant mode frequencies, $Q_{\pi}$ and $Q_{0}$ are the loaded Qs, and $A_{\pi}$ and $A_{0}$ are mode amplitudes. The latter depend on probe gain, so it is primarily their ratio that is of interest. The model requires probe gain to be small (so as not to modify cavity fields) and constant over the $6 \mathrm{MHz}$ measurement range. The (absolute) value for $A_{\pi}$ turned out to be $0.00224(-53.0 \mathrm{~dB})$ for the half-cell probe, and $0.00624(-44.1 \mathrm{~dB})$ for the full-cell probe. During high-power operation it was subsequently found that the full-cell signal was too large, as it gradually damaged a medium-power attenuator (50 $\mathrm{W}$ average, $2 \mathrm{~kW}$ peak) at the probe output even though the attenuator was rated for the applied power. Thus, the ideal probe response is about $-53 \pm 2 \mathrm{~dB}$. 

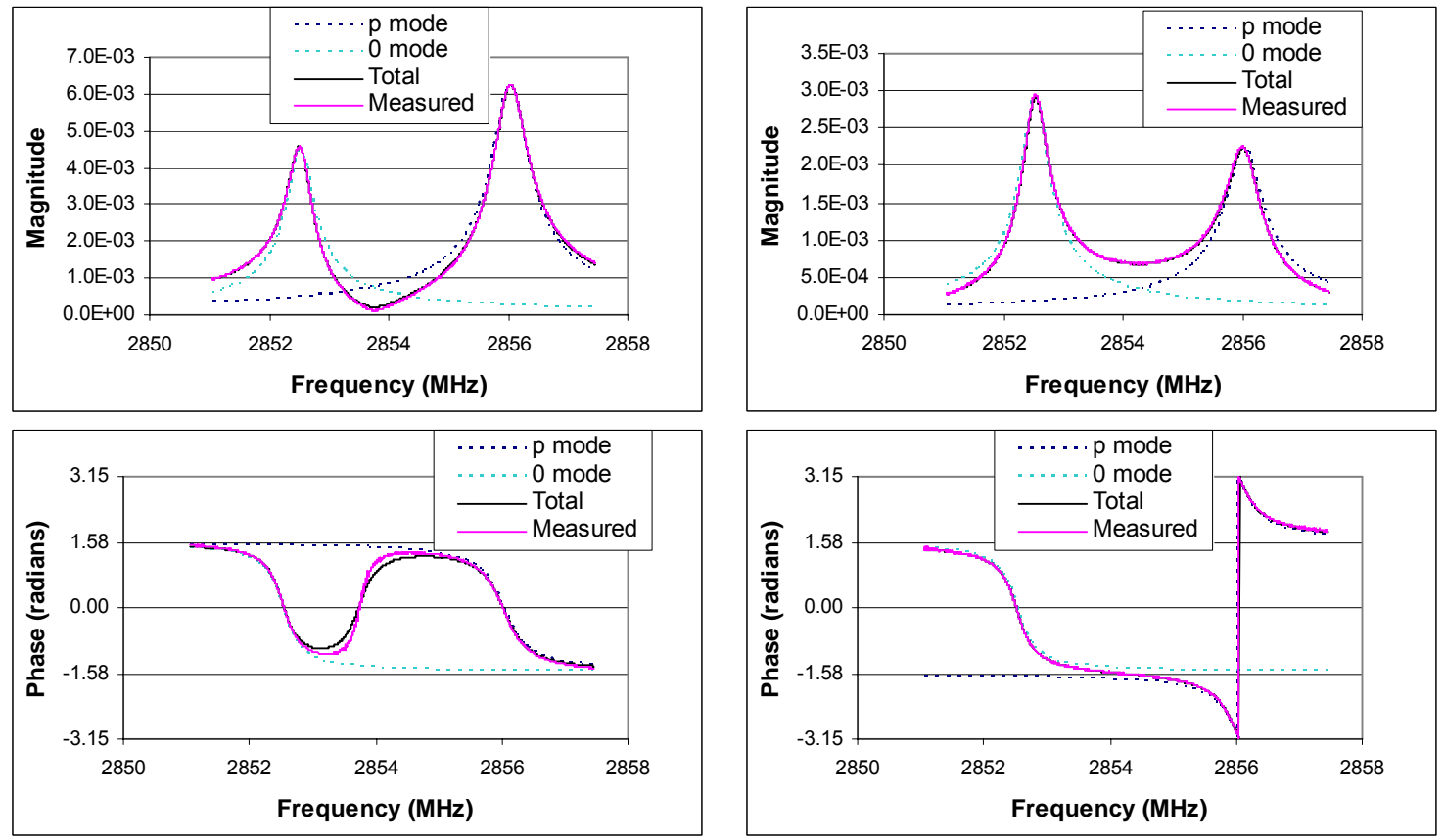

Full-cell probe

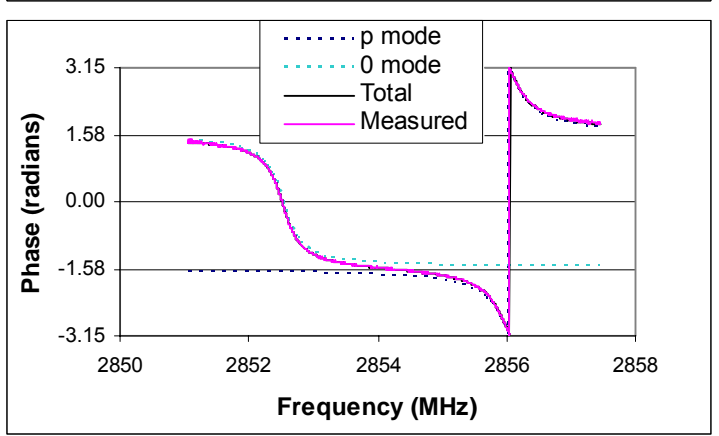

Half-cell probe

Figure $10 \mathrm{~S}_{21}$ amplitude and phase, with port 2 connected to the full cell (left) and half cell (right). The solid red line is measured data, and the dashed and solid black lines are generated from Eq. (2) after fitting for model parameters.

\begin{tabular}{|l|c|c|}
\hline Parameter & $\pi$ mode & 0 mode \\
\hline$A$ (half-cell probe) & -1 & +1.31 \\
\hline$A$ (full-cell probe) & +1 & +0.72 \\
\hline$f$ (resonant frequency, $\mathrm{MHz}$ ) & 2856.03 & 2852.53 \\
\hline$Q$ (loaded quality factor) & 5160 & 6980 \\
\hline$\tau$ (time constant $Q / \pi f, \mathrm{~ns})$ & 576 & 779 \\
\hline$\beta$ (coupling coefficient) & 1.34 & 0.68 \\
\hline
\end{tabular}

Table 1 Best-fit parameters and derived quantities. Mode amplitudes $A$ have been normalized to the absolute value of the $\pi$-mode amplitude for each probe. The $\beta$ values are actually derived from reflection measurements, and are equal to the VSWR for the overcoupled $\pi$ mode, and 1/VSWR for the undercoupled zero mode.

Best-fit values for the model parameters of Eq. 2 are given in Table 1, and the curves from the model are displayed in Fig. 10. Mode amplitudes in the table are normalized to the (absolute) value of the $\pi$ mode (for each probe) in order to eliminate probe gain. The normalized electric fields $E_{z}$ on the axis of the gun as computed by Superfish for the balanced gun are shown in Fig. 11. The curves represent standing-wave amplitudes. Evaluated at the cathode surface the mode ratio $E_{0} / E_{\pi}=-1.28$, and evaluated at the standing-wave maximum in the full cell $E_{0} / E_{\pi}=0.72$. These values agree well with the normalized mode amplitudes of Table 1, and indicate that probe gain is largely constant over this range of interest. 


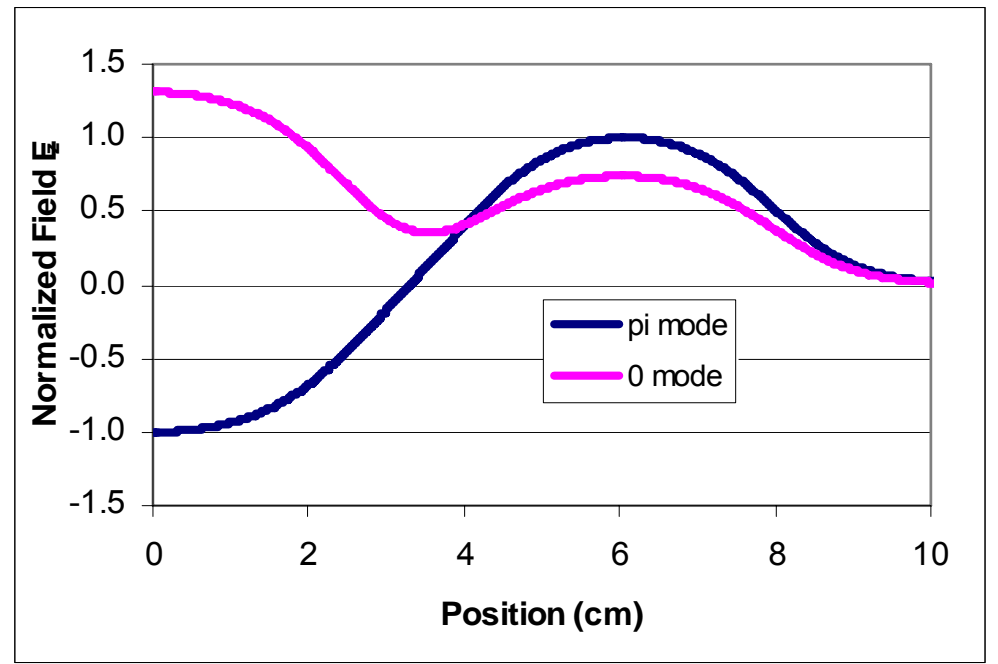

Figure 11 Normalized electric field on axis of field-balanced gun, as computed by Superfish.

With the help of Superfish, a probe can be can be calibrated to measure the electric field at a particular point in the cavity by relating the field amplitude there to the total stored energy. In particular, the square of a standing-wave maximum $E_{a}$ on the axis of the gun is proportional to the stored energy $U$ as indicated in Eq. (3). The stored energy is related to the power lost on the structure walls as shown in Eq. (4), where $Q_{L}$ is the loaded quality factor, $\beta$ is the coupling coefficient, and $\omega / 2 \pi$ is the resonant frequency. (Here we assume a single mode, to which all quantities refer, and that the gun is driven at the resonant frequency.) The power lost in the walls is related to the power incident in the waveguide and the derivative of the stored energy as shown in Eq. (5). At steady state the ratio of probe power to incident power is equal to the square of the mode amplitude $A$ (i.e., $\left|\mathrm{S}_{21}\right|$, as measured by the probe), as in Eqs. (2) and (6). Substituting Eqs. (4-6) into Eq. (3), the steady state field $E_{a}$ is given by Eq. (7). Since the probes are simple antennas, the power at the probe is always proportional to the square of the field, Eq. (8). Thus Eq. (7) which was derived for steady state is actually valid at all times.

$$
\begin{gathered}
E_{a}^{2} \equiv \frac{U}{\alpha} \\
U=\frac{Q_{L}(\beta+1) P_{\text {wall }}}{\omega} \\
P_{\text {wall }}(t)=P_{\text {incident }}-P_{\text {reflected }}-\frac{d U}{d t}=\frac{4 \beta}{(\beta+1)^{2}} P_{\text {incident }}-\frac{d U}{d t} \\
P_{\text {probe }}=A^{2} P_{\text {incident }} \\
E_{a}=\sqrt{\frac{4 \beta Q_{L}}{\omega \alpha(\beta+1)} \frac{P_{\text {probe }}}{A^{2}}} \\
E_{a}^{2}(t) \propto P_{\text {probe }}(t)
\end{gathered}
$$




\begin{tabular}{|l|c|c|}
\hline Parameter & $\pi$ mode & 0 mode \\
\hline$U(\mathrm{~J})$ & 0.2813 & 0.2588 \\
\hline$E_{a}(\mathrm{MV} / \mathrm{m})$ & 24.67 & 31.62 \\
\hline$\alpha\left(\mathrm{J} /(\mathrm{MV} / \mathrm{m})^{2}\right)$ & $4.6210^{-4}$ & $2.5910^{-4}$ \\
\hline
\end{tabular}

Table 2. Superfish values for total stored energy and half-cell standing-wave maxima for the $\pi$-mode balanced gun. The data yields $\alpha$, the constant of proportionality in Eq. (3).

Superfish values for total stored energy and half-cell standing-wave maxima are given in Table 2. The computations are for a gun tuned for field balance, driven at the $\pi$ and zero-mode frequencies (one at a time). The derivation of Eq. (7) is technically correct for a single mode only, but due to mode separation the contribution from the zero mode at the $\pi$-mode resonance is small and typically ignored. It leads to a few percent error in measured field value. The mode-beating effect is, however, clearly visible on the transient signal at high power.

\section{Acknowledgements}

The authors would like to acknowledge multiple discussions with D.H. Dowell and J.E. Clendenin. Also we appreciate the assistance of M. Nalls at SSRL. Without his assistance this work would have been impossible.

\section{References}

${ }^{1}$ L. C. Maier and J. C. Slater, "Field Strength Measurements in Resonant Cavities", Journal of Applied Physics, Vol. 23 No. 1, pp. 68-77 (1952).

${ }^{2}$ E. L. Ginzton, Microwave Measurements, (McGraw-Hill, 1957), p. 139.

${ }^{3}$ For non-resonant perturbative measurement, see S.M. Hanna et. al., "Microwave Cold-Testing Techniques for the NLC", SLAC-PUB-9901, and references therein.

${ }^{4}$ J. H. Billen and L. M. Young, "POISSON SUPERFISH", Los Alamos National Laboratory report LA-UR-96-1834. 